\title{
Extremal numbers of cycles revisited
}

\author{
David Conlon*
}

\begin{abstract}
We give a simple geometric interpretation of an algebraic construction of Wenger that yields $n$-vertex graphs with no cycle of length 4, 6 or 10 and close to the maximum number of edges.
\end{abstract}

What is the maximum number of edges in an $n$-vertex graph containing no cycle $C_{2 k}$ of length $2 k$ ? This is one of the most closely studied yet elusive problems in combinatorics. Despite many decades of intense interest in this and related problems in extremal graph theory [11, the answer is only reasonably well understood for cycles of length 4,6 and 10 .

If we write $\operatorname{ex}(n, H)$ for the maximum number of edges in an $n$-vertex graph containing no copy of the graph $H$, then a result attributed to Erdös [2, [5] says that $\operatorname{ex}\left(n, C_{2 k}\right) \leq C n^{1+1 / k}$ for some constant $C$ depending only on $k$. For $C_{4}$, a matching lower bound, due to Klein, already appeared in a paper of Erdős [4 from 1938. For $C_{6}$ and $C_{10}$, constructions matching the upper bound were found by Benson [1] and Singleton [9] in 1966, though several alternative constructions have since been found [7, 8, 13]. None of these constructions can be described as simple, though that of Wenger [13] has the best claim. Here we show that this claim is justified, by rephrasing his algebraic construction in geometric terms that make its properties manifest.

Let $q$ be a prime power and $\mathbb{F}_{q}$ the finite field of order $q$. For each $x \in \mathbb{F}_{q}^{k}$ and $z \in \mathbb{F}_{q}$, we form the line

$$
\left\{x+y \cdot\left(1, z, z^{2}, \ldots, z^{k-1}\right): y \in \mathbb{F}_{q}\right\} .
$$

The number of distinct lines of this form is exactly $q^{k}$, since there are $q$ distinct directions, determined by the different values for $z$, and exactly $q^{k-1}$ parallel lines in each direction. We form a bipartite graph $D_{k}(q)$ between two sets $P$ and $L$ of order $q^{k}$, where the vertices of $P$ are indexed by the points of $\mathbb{F}_{q}^{k}$, the vertices of $L$ are indexed by the $q^{k}$ lines described above and there is an edge between $p \in P$ and $\ell \in L$ if and only if $p \in \ell$. Our main result is as follows.

Theorem 1. For $k=2,3$ and $5, D_{k}(q)$ is a $C_{2 k}$-free bipartite graph between sets $P$ and $L$ of order $n=q^{k}$ with $n^{1+1 / k}$ edges.

Proof. The bound on the number of edges follows from the fact that each of the $q^{k}$ lines contains exactly $q$ points, so there are $q^{k+1}=n^{1+1 / k}$ edges in total. Note now that any cycle in $D_{k}(q)$ is of the form $p_{1} \ell_{1} p_{2} \ell_{2} \ldots p_{t} \ell_{t} p_{1}$, where $p_{i} \in P$ and $\ell_{i} \in L$ for all $1 \leq i \leq t$. We make two simple observations about these cycles:

- For any $1 \leq i \leq t($ taken $\bmod t), \ell_{i}$ and $\ell_{i+1}$ cannot be parallel.

*Department of Mathematics, California Institute of Technology, Pasadena, CA 91125. Email: dconlon@caltech.edu. 
- If $t \leq k$, then, for any $1 \leq i \leq t$, there must be another line $\ell_{i^{\prime}}$ with $1 \leq i^{\prime} \leq t$ parallel to $\ell_{i}$.

The first observation is obvious, since two parallel lines, both passing through the point $p_{i+1}$, must coincide. For the second observation, note that if $\ell_{i}$ has direction determined by $z_{i}$ for each $1 \leq i \leq t$, then the difference $p_{i+1}-p_{i}$ is a non-zero multiple of $\left(1, z_{i}, z_{i}^{2}, \ldots, z_{i}^{k-1}\right)$. Adding over all $i$, we have that

$$
0=\sum_{i=1}^{t}\left(p_{i+1}-p_{i}\right)=\sum_{i=1}^{t} a_{i}\left(1, z_{i}, z_{i}^{2}, \ldots, z_{i}^{k-1}\right)
$$

for some collection of non-zero coefficients $a_{i}$. But it is a well-known fact, proved by considering the Vandermonde determinant, that any $k$ distinct vectors of the form $\left(1, z, z^{2}, \ldots, z^{k-1}\right)$ are linearly independent. Hence, for the sum $\sum_{i=1}^{t} a_{i}\left(1, z_{i}, z_{i}^{2}, \ldots, z_{i}^{k-1}\right)$ to be zero, each direction must appear at least twice. That is, every line $\ell_{i}$ has at least one parallel line $\ell_{i^{\prime}}$. We can now dispense with each case as a bulletpoint:

- No $D_{k}(q)$ with $k \geq 2$ contains a cycle of length 4 , since any such cycle $p_{1} \ell_{1} p_{2} \ell_{2} p_{1}$ must be such that $\ell_{1}$ and $\ell_{2}$ are both parallel and not parallel by the two observations above.

- No $D_{k}(q)$ with $k \geq 3$ contains a cycle of length 6 , since any such cycle $p_{1} \ell_{1} p_{2} \ell_{2} p_{3} \ell_{3} p_{1}$ must again be such that the three lines $\ell_{1}, \ell_{2}$ and $\ell_{3}$ are all parallel and all not parallel by our two observations.

- No $D_{k}(q)$ with $k \geq 5$ contains a cycle of length 10 . Indeed, any such cycle $p_{1} \ell_{1} p_{2} \ell_{2} p_{3} \ell_{3} p_{4} \ell_{4} p_{5} \ell_{5} p_{1}$ must have one group of two parallel lines and another group of three parallel lines. But then it is impossible to enforce the condition that $\ell_{i}$ not be parallel to $\ell_{i+1}$ for all $1 \leq i \leq 5$ (taken mod $5)$.

Suppose now that $\theta_{k, \ell}$ is the graph consisting of $\ell$ internally disjoint paths of length $k$, each with the same endpoints. In particular, $\theta_{k, 2}$ is just $C_{2 k}$, so the problem of determining ex $\left(n, \theta_{k, \ell}\right)$ extends the problem of determining $\operatorname{ex}\left(n, C_{2 k}\right)$. The extremal numbers for these theta graphs were first studied by Faudree and Simonovits [6], who proved that $\operatorname{ex}\left(n, \theta_{k, \ell}\right) \leq C n^{1+1 / k}$ for a constant $C$ depending only on $k$ and $\ell$. On the other hand, a result of Conlon [3] says that for any $k$ there exists a natural number $\ell$ and a positive constant $c$ such that $\operatorname{ex}\left(n, \theta_{k, \ell}\right) \geq c n^{1+1 / k}$.

The following question therefore becomes valid: for each $k$, what is the smallest $\ell$ such that $\operatorname{ex}\left(n, \theta_{k, \ell}\right) \geq$ $c n^{1+1 / k}$ for some positive $c$ ? The results on $\operatorname{ex}\left(n, C_{2 k}\right)$ show that for $k=2,3$ or 5 we can take $\ell=2$. A much more recent result of Verstraëte and Williford [12] says that for $k=4$ it suffices to take $\ell=3$. As a bonus, we show that the graph $D_{4}(q)$ has a one-sided version of the property satisfied by the Verstraëte-Williford graph, thus giving a natural interpolation between the cases $k=3$ and 5 .

Theorem 2. $D_{4}(q)$ is a bipartite graph between sets $P$ and $L$ of order $n=q^{4}$ with $n^{5 / 4}$ edges such that any two vertices in $P$ have at most 2 paths of length 4 between them.

Proof. A theta graph $\theta_{4, \ell}$ whose endpoints are $p, p^{\prime} \in P$ consists of paths $p \ell_{1, j} p_{2, j} \ell_{2, j} p^{\prime}$ with $1 \leq j \leq \ell$. However, any two such paths yield a cycle, so we can still apply our basic observations:

- No $D_{k}(q)$ with $k \geq 4$ contains a theta graph $\theta_{4,3}$ with both endpoints $p, p^{\prime} \in P$. Indeed, any such graph consists of paths $p \ell_{1, j} p_{2, j} \ell_{2, j} p^{\prime}$ for $1 \leq j \leq 3$ where $\ell_{1, j}$ and $\ell_{2, j}$ are not parallel, 
by our first observation. But, since $p \ell_{1,1} p_{2,1} \ell_{2,1} p^{\prime} \ell_{2,2} p_{2,2} \ell_{1,2} p$ is a cycle, we must have, by our second observation, that $\ell_{1,1}$ is parallel to $\ell_{2,2}$ and $\ell_{2,1}$ is parallel to $\ell_{1,2}$. Similarly, considering the cycle $p \ell_{1,1} p_{2,1} \ell_{2,1} p^{\prime} \ell_{2,3} p_{2,3} \ell_{1,3} p$, we must have that $\ell_{1,1}$ is parallel to $\ell_{2,3}$ and $\ell_{2,1}$ is parallel to $\ell_{1,3}$. But then the cycle $p \ell_{1,2} p_{2,2} \ell_{2,2} p^{\prime} \ell_{2,3} p_{2,3} \ell_{1,3} p$ violates the first observation, since $\ell_{2,2}$ and $\ell_{2,3}$ are parallel.

We conclude with a conjecture. It is now quite commonplace to believe that the true value of certain extremal numbers lie below what the classical arguments give. We suspect that this should already be the case for $C_{8}$, that is, that $\operatorname{ex}\left(n, C_{8}\right)=o\left(n^{5 / 4}\right)$. Proving this is likely to be exceptionally difficult, but a first step might be to show that no construction of the type studied in this paper can yield the lower bound. Concretely, we have the following conjecture. The definition of a line in $\mathbb{F}_{q}^{k}$ agrees with that used throughout, namely, a set of the form $\left\{x+y \cdot z: y \in \mathbb{F}_{q}\right\}$ for some $x, z \in \mathbb{F}_{q}^{k}$.

Conjecture 1. The maximum number of lines in $\mathbb{F}_{q}^{4}$ containing no $C_{4}$ of lines, that is, four distinct lines $\ell_{1}, \ell_{2}, \ell_{3}, \ell_{4}$ such that $\ell_{i}$ and $\ell_{i+1}$ intersect in distinct points for all $1 \leq i \leq 4$ (taken mod 4 ), is $o\left(q^{4}\right)$.

A similar conjecture can be made for any cycle of length at least 6 .

Note added. After this article was accepted, Tibor Szabó drew my attention to his lecture notes [10], where Wenger's construction is also described in very similar terms.

\section{References}

[1] C. T. Benson, Minimal regular graphs of girths eight and twelve, Canad. J. Math. 18 (1966), 1091-1094.

[2] J. A. Bondy and M. Simonovits, Cycles of even length in graphs, J. Combin. Theory Ser. B 16 (1974), 97-105.

[3] D. Conlon, Graphs with few paths of prescribed length between any two vertices, Bull. Lond. Math. Soc. 51 (2019), 1015-1021.

[4] P. Erdős, On sequences of integers no one of which divides the product of two others and on some related problems, Mitt. Forsch.-Inst. Math. Mech. Univ. Tomsk 2 (1938), 74-82.

[5] P. Erdős, Extremal problems in graph theory, in Theory of Graphs and its Applications (Proc. Sympos. Smolenice, 1963), 29-36, Publ. House Czechoslovak Acad. Sci., Prague, 1964.

[6] R. J. Faudree and M. Simonovits, On a class of degenerate extremal graph problems, Combinatorica 3 (1983), 83-93.

[7] F. Lazebnik and V. A. Ustimenko, Explicit construction of graphs with an arbitrary large girth and of large size, Discrete Appl. Math. 60 (1995), 275-284.

[8] K. E. Mellinger and D. Mubayi, Constructions of bipartite graphs from finite geometries, J. Graph Theory 49 (2005), 1-10.

[9] R. R. Singleton, On minimal graphs of maximum even girth, J. Combin. Theory 1 (1966), 306-332. 
[10] T. Szabó, lecture notes available at http://discretemath.imp.fu-berlin.de/DMIII-2018/notesevencycles.pdf.

[11] J. Verstraëte, Extremal problems for cycles in graphs, in Recent trends in combinatorics, 83-116, IMA Vol. Math. Appl., 159, Springer, Cham, 2016.

[12] J. Verstraëte and J. Williford, Graphs without theta subgraphs, J. Combin. Theory Ser. B 134 (2019), 76-87.

[13] R. Wenger, Extremal graphs with no $C^{4}$ 's, $C^{6}$ 's, or $C^{10}$ 's, J. Combin. Theory Ser. B 52 (1991), $113-116$. 